\title{
Semi-blind Channel Estimation and Data Detection for OFDM Systems over Frequency-Selective Fading Channels
}

\author{
Tao Cui and Chintha Tellambura \\ Department of Electrical and Computer Engineering \\ University of Alberta \\ Edmonton, AB, Canada T6G 2V4 \\ Email: \{taocui, chintha\}@ece.ualberta.ca
}

\begin{abstract}
This paper considers semi-blind channel estimation and data detection for OFDM systems over frequency-selective fading channels. Using the maximum likelihood (ML) principle, we derive a blind channel estimator by taking the time domain transmitted signal as Gaussian (due to the central limit theorem) and averaging the likelihood function over the resulting Gaussian distribution. This estimator is realized using the steepest descent algorithm. Similarly, our semi-blind data detector integrates the channel impulse response (CIR) out of the likelihood function, which is realized using sphere decoding and V-BLAST. Simulation results show that our proposed channel estimator and data detector perform a fraction of $\mathrm{dB}$ within an ideal reference receiver.
\end{abstract}

\section{INTRODUCTION}

Orthogonal Frequency Division Multiplexing (OFDM) is a promising candidate for high-data-rate wireless communications due to its high spectral efficiency, robustness to frequency selective fading and simple equalization. Coherent detection requires reliable estimation of the channel impulse response (CIR). In practical OFDM systems, pilot symbolaided channel estimation for OFDM has been widely used [1], [2]. However, the cyclic prefix (CP) and pilots together constitute a significant bandwidth loss, which has motivated the development of blind equalization methods.

Blind channel estimators that exploit the CP-induced redundancy and pilot subcarriers are developed in [3]. Reference [4] develops a blind subspace approach exploiting CP-induced cyclostationarity. A blind channel estimator by exploiting the finite alphabet property of modulation symbols is proposed in [5]. Subspace-based blind channel estimator using virtual carriers has been derived in [6]. In [7], a sufficient condition for blind channel estimation is given.

In wireless environments, the user's mobility fluctuates the multipath channel. Non-negligible fluctuations of the CIR are expected between consecutive OFDM symbols (or even within each frame). Hence, the subspace based blind channel estimators [3]-[6], which need several OFDM symbols to identify the channel, may not be practical and moreover, they have high complexity. In this paper, we present a semi-blind channel estimator and a semi-blind data detector. Importantly, our estimators need just one OFDM symbol. Assuming that the transmitted time domain samples can be modelled as a complex Gaussian process, we derive the semi-blind channel estimator by averaging the likelihood function over the complex Gaussian distribution. The resulting channel estimator can be realized using the steepest descent algorithm. On the other hand, averaging the likelihood function over the CIR gives the semi-blind data detector as a discrete integer least squares (LS) optimization problem. Since exhaustive search is computationally prohibitive, we use both sphere decoding (SD) [8] and vertical-bell labs layered space-time (V-BLAST) [9] to solve this quadratic optimization problem.

The rest of the paper is organized as follows. Section II reviews the basic baseband OFDM system model. Section III introduces the semi-blind channel estimator. In Section IV, we derive the semi-blind data detector. Furthermore, SD and VBLAST algorithms to solve the integer LS problem are also presented. Section V gives computer simulation results and Section VI concludes the paper.

Notation: Bold symbols denote matrices or vectors. $(\cdot)^{*}$, $(\cdot)^{T}$, and $(\cdot)^{H}$ denote conjugate, transpose and conjugate transpose respectively. $\operatorname{Re}(\mathbf{x})$ denotes the real part of $\mathbf{x}$. If $x$ and $y$ are Gaussian random variables with $E[x]=\mu_{x}$, $E[y]=\mu_{y}$ and $E\left[\left(x-\mu_{x}\right)^{2}\right]=E\left[\left(y-\mu_{y}\right)^{2}\right]=\sigma^{2} / 2$, $z=x+j y$ (where $j=\sqrt{-1}$ ) is a complex Gaussian random variable (CGRV) and is denoted by $z \sim \mathcal{C N}\left(\mu_{x}+j \mu_{y}, \sigma^{2}\right)$. The discrete Fourier transform (DFT) matrix of size $N \times N$ is given by $\mathbf{F}=1 / \sqrt{N}\left[e^{-j \frac{2 \pi}{N} k l}\right], k, l \in 0,1, \cdots, N-1$. $\mathbf{A}_{D}$ denotes a diagonal matrix whose diagonal terms are components of vector $\mathbf{a}$.

\section{OFDM BASEBAND MODEL}

In an OFDM system, the source data are grouped and mapped into the symbols from a constellation $\mathcal{Q}$, which are modulated by inverse DFT (IDFT) on $N$ parallel subcarriers. Note that $X_{k}, k=0,1, \ldots, N-1$ are called OFDM input symbols, where

$$
X_{k}= \begin{cases}d_{k} & k \in I_{d} \\ p_{k} & k \in I_{p}\end{cases}
$$


$I_{d}$ is the index set of data subcarriers with $N_{d}$ elements and $I_{p}$ is the index set of subcarriers reserved for pilot symbols with $N_{p}$ elements. Pilot symbols $X_{k}, k \in I_{p}$, are known a priori at the receiver (hence the name pilot symbols). We have $N_{d}+N_{p}=N$. The average energy of $X_{k}$ is normalized to unity with $E\left\{\left|X_{k}\right|^{2}\right\}=1$. The term "OFDM symbol" is used to denote the entire IDFT output $\left\{x_{0}, x_{1}, \cdots, x_{N-1}\right\}$. The input symbol duration is $T_{s}$ and the OFDM symbol duration is $N T_{s}$. These samples are appropriately pulse shaped to construct the time domain signal $x(t)$ for transmission. We assume that the composite CIR which includes transmit and receive pulse shaping and the physical channel response between the transmitter and receiver may be modelled as

$$
h(\tau)=\sum_{l=0}^{L-1} h_{l} \delta\left(\tau-\tau_{l}\right)
$$

where $h_{l} \sim \mathcal{C N}\left(0, E\left[h_{l}^{2}\right]\right)$ and $\tau_{l}$ is the delay of the lth tap. Typically, it is assumed that $\tau_{l}=l T_{s}$ and this results in a finite impulse response filter with an effective length $L$. Assuming that the channel remains constant during each OFDM symbol, but it varies between OFDM symbols, and the cyclic prefix is sufficiently long $\left(N_{g}>L\right)$, the post-DFT received samples $Y_{k}$ are given as

$$
\begin{aligned}
Y_{k} & =H_{k} X_{k}+W_{k}, \quad 0 \leq k \leq N-1 \\
& =\frac{1}{\sqrt{N}} \sum_{n=0}^{N} H_{k} x_{n} e^{-j 2 \pi k n / N}+W(k)
\end{aligned}
$$

where $H_{k}=H(j 2 \pi k / N)$ is the complex channel frequency response at subcarrier $k, H(j \omega)$ is the Fourier transform of the CIR and $W_{k}, k=0,1, \cdots, N-1$ are independent and identically distributed (i.i.d) CGRV's with zero mean and variance $\sigma_{n}^{2}$. Assuming $\tau_{l}=l T_{s}$, we find $\mathbf{H}=\mathbf{F}_{L} \mathbf{h}$, where $\mathbf{H}=\left[H_{0}, H_{1}, \cdots, H_{N-1}\right]^{T}, \mathbf{h} \in \mathcal{C}^{L}$ is the CIR and $\mathbf{F}_{L}$ is a $N \times L$ submatrix of the DFT matrix $\mathbf{F}$. We can vectorize (3) as

$$
\mathbf{Y}=\mathbf{X}_{D} \mathbf{F}_{L} \mathbf{h}+\mathbf{W}
$$

or equivalently

$$
\mathbf{Y}=\mathbf{H}_{D} \mathbf{F} \mathbf{x}+\mathbf{W}
$$

where $\mathbf{X}_{D}=\operatorname{diag}\left\{X_{0}, \cdots, X_{N-1}\right\}$ and $\mathbf{H}_{D}=$ $\operatorname{diag}\left\{H_{0}, \cdots, H_{N-1}\right\}$ are diagonal matrices, $\mathbf{x}=$ $\left[x_{0}, \cdots, x_{N-1}\right]^{T}$ and $\mathbf{Y}=\left[Y_{0}, \ldots, Y_{N-1}\right]^{T}$.

\section{SEMI-BLIND CHANNEL ESTIMATION}

The received symbol vector $\mathbf{Y}(5)$ is Gaussian with mean $\mathbf{H}_{D} \mathbf{F x}$ and covariance matrix $\sigma_{n}^{2} \mathbf{I}_{N}$. The likelihood function for the unknown parameters $\mathbf{x}$ and $\mathbf{H}_{D}$ is given by

$$
f\left(\mathbf{Y} \mid \mathbf{x}, \mathbf{H}_{D}\right)=\exp \left\{-\frac{1}{\sigma_{n}^{2}}\left\|\mathbf{Y}-\mathbf{H}_{D} \mathbf{F} \mathbf{x}\right\|^{2}\right\} .
$$

The time domain transmitted signal $x_{n}$ can be modelled as complex Gaussian via the central limit theorem when $N$ is large [10]. In blind channel estimation, there always exists a phase ambiguity, which can be solved by inserting pilot symbols. If pilot symbols are inserted, the mean of $x_{n}$ is non-zero. It can be readily verified that the covariance matrix and mean of $\mathbf{x}$ are given by

$$
\overline{\mathbf{x}}=E\{\mathbf{x}\}=\mathbf{F}^{H} \mathbf{X}_{p}, E\left\{(\mathbf{x}-\overline{\mathbf{x}})(\mathbf{x}-\overline{\mathbf{x}})^{H}\right\}=\mathbf{F}^{H} \boldsymbol{\Lambda}_{d} \mathbf{F}
$$

where

$$
\mathbf{X}_{p}(k)=\left\{\begin{array}{cc}
p_{k} & k \in I_{p} \\
0 & k \notin I_{p}
\end{array}, \boldsymbol{\Lambda}_{d}(k, k)=\left\{\begin{array}{cc}
1 & k \in I_{d} \\
0 & k \notin I_{d}
\end{array} .\right.\right.
$$

The average of $f\left(\mathbf{Y} \mid \mathbf{x}, \mathbf{H}_{D}\right)$ with respect to $\mathbf{x}$ gives the marginal likelihood function $f\left(\mathbf{Y} \mid \mathbf{H}_{D}\right)$, which can be expressed as

$$
f\left(\mathbf{Y} \mid \mathbf{H}_{D}\right)=\int f\left(\mathbf{Y} \mid \mathbf{x}, \mathbf{H}_{D}\right) p(\mathbf{x}) d \mathbf{x}
$$

where $p(\mathbf{x})$ is the pdf of $\mathbf{x}$. We can evaluate (9) as

$$
\begin{aligned}
& f\left(\mathbf{Y} \mid \mathbf{H}_{D}\right)=\frac{1}{\operatorname{det}\left(\sigma_{n}^{2}+\boldsymbol{\Lambda}_{d} \mathbf{H}_{D}^{H} \mathbf{H}_{D}\right)} \\
& \times \exp \left\{-\left[\mathbf{X}_{p}^{H}\left(\boldsymbol{\Lambda}_{d}+\sigma_{n}^{2}\left(\mathbf{H}_{D}^{H} \mathbf{H}_{D}\right)^{-1}\right)^{-1} \mathbf{X}_{p}\right.\right. \\
& +\sigma_{n}^{2} \mathbf{Y}^{H}\left(\mathbf{H}_{D}^{H} \mathbf{H}_{D} \boldsymbol{\Lambda}_{d}+\sigma_{n}^{2} \mathbf{I}_{N}\right)^{-1} \mathbf{Y} \\
& \left.\left.+2 \sigma_{n}^{2} \operatorname{Re}\left(\mathbf{Y}^{H} \mathbf{H}_{D}\left(\boldsymbol{\Lambda}_{d} \mathbf{H}_{D}^{H} \mathbf{H}_{D}+\sigma_{n}^{2} \mathbf{I}_{N}\right)^{-1} \mathbf{X}_{p}\right)\right]\right\} .
\end{aligned}
$$

After some manipulations, the log likelihood function $\Lambda\left(\mathbf{Y} \mid \mathbf{H}_{D}\right)=\ln f\left(\mathbf{Y} \mid \mathbf{H}_{D}\right)$ can be simplified to

$$
\begin{aligned}
\Lambda\left(\mathbf{Y} \mid \mathbf{H}_{D}\right)= & -\sigma_{n}^{2} \sum_{k \in I_{v}}\left(\frac{\left|Y_{k}\right|^{2}}{\left|H_{k}\right|^{2}+\sigma_{n}^{2}}+\ln \left(\left|H_{k}\right|^{2}+\sigma_{n}^{2}\right)\right) \\
& -\sum_{k \in I_{p}}\left|Y_{k}-H_{k} X_{k}\right|^{2} .
\end{aligned}
$$

Maximizing (11) is equivalent to minimizing

$$
\begin{aligned}
g(\mathbf{h})= & \sigma_{n}^{2} \sum_{k \in I_{v}}\left(\frac{\left|Y_{k}\right|^{2}}{\left|H_{k}\right|^{2}+\sigma_{n}^{2}}+\ln \left(\left|H_{k}\right|^{2}+\sigma_{n}^{2}\right)\right) \\
& +\sum_{k \in I_{p}}\left|Y_{k}-H_{k} X_{k}\right|^{2} .
\end{aligned}
$$

Since $H_{k}=\sum_{l=1}^{L} h_{l} e^{-2 \pi l k / N}$, the CIR $\mathbf{h}$ can be estimated using (12) via the steepest descent algorithm. Note that if no pilot exists, (12) reduces to the semi-blind estimator

$$
g(\mathbf{h})=\sum_{k=0}^{N-1}\left(\frac{\left|Y_{k}\right|^{2}}{\left|H_{k}\right|^{2}+\sigma_{n}^{2}}+\ln \left(\left|H_{k}\right|^{2}+\sigma_{n}^{2}\right)\right) .
$$

We can readily see that $\mathbf{h}$ and $\mathbf{h} e^{j \theta}$ both are possible solutions of (13), where $\theta$ is an arbitrary phase shift. This indicates a problem of phase ambiguity. The resolution of such ambiguity requires the use of pilot symbols. If $H_{k}$ 's are independent, the solution of (13) is $\left|Y_{k}\right|^{2}=\left|H_{k}\right|^{2}+\sigma_{n}^{2}$.

On the other hand, if sufficient pilots are available, the channel can be estimated differently. Assuming that $N_{p}$ pilots 
are inserted $\left(N_{p} \geq L\right)$, the channel can be initially estimated via minimum mean square error (MMSE) estimator [1] as

$$
\hat{\mathbf{h}}=\left(\mathbf{F}_{p}^{H} \mathbf{P}_{D}^{H} \mathbf{P}_{D} \mathbf{F}_{p}+\sigma_{n}^{2} \mathbf{R}_{h}^{-1}\right)^{-1} \mathbf{F}_{p}^{H} \mathbf{P}_{D}^{H} \mathbf{Y}_{p}
$$

where $\mathbf{P}_{D}=\operatorname{diag}\left\{X_{I_{p}(1)}, \ldots, X_{I_{p}\left(N_{p}\right)}\right\}, \mathbf{F}_{p}$ is the corresponding $N_{p} \times L$ submatrix of $\mathbf{F}$ and $\mathbf{R}_{h}=E\left\{\mathbf{h} \mathbf{h}^{H}\right\}$ is the covariance matrix of $\mathbf{h}$. If $N_{p}<L$, the channel can be estimated by using generalized Schur decomposition. When all the symbols are pilots, (13) reduces to a least squares (LS) estimator, which is maximum likelihood. Therefore, our semiblind estimator (12) can be viewed as an enhanced LS channel estimator.

After the channel is estimated using (12), one-tap equalization can be used to recover the transmitted data symbols. Note that (12) is applicable for any constellations.

\section{Semi-Blind Data Detection}

\section{A. Semi-blind data detector}

Similarly, the received symbol vector $\mathbf{Y}$ is Gaussian with mean $\mathbf{X}_{D} \mathbf{F}_{L} \mathbf{h}$ and covariance matrix $\sigma_{n}^{2} \mathbf{I}_{N}$ using (4). The likelihood function for the unknown parameters $\mathbf{h}$ and $\mathbf{X}_{D}$ is given by

$$
f\left(\mathbf{Y} \mid \mathbf{h}, \mathbf{X}_{D}\right)=\exp \left\{-\frac{1}{\sigma_{n}^{2}}\left\|\mathbf{Y}-\mathbf{X}_{D} \mathbf{F}_{L} \mathbf{h}\right\|^{2}\right\} .
$$

We assume $\mathbf{h}$ is a complex Gaussian vector (Rayleigh fading) with zero mean and covariance matrix $\mathbf{R}_{h}$. The average of $f\left(\mathbf{Y} \mid \mathbf{h}, \mathbf{X}_{D}\right)$ with respect to $\mathbf{h}$ gives the marginal likelihood function $f\left(\mathbf{Y} \mid \mathbf{X}_{D}\right)$, which can be expressed as

$$
f\left(\mathbf{Y} \mid \mathbf{X}_{D}\right)=\int f\left(\mathbf{Y} \mid \mathbf{h}, \mathbf{X}_{D}\right) p(\mathbf{h}) d \mathbf{h}
$$

where $p(\mathbf{h})$ is the pdf of $\mathbf{h}$. We evaluate (16) to give

$$
\begin{aligned}
& f\left(\mathbf{Y} \mid \mathbf{X}_{D}\right)=\frac{1}{\operatorname{det}\left(\sigma_{n}^{2} \mathbf{I}_{N}+\mathbf{F}_{L}^{H} \mathbf{X}_{D}^{H} \mathbf{X}_{D} \mathbf{F}_{L}\right)} \\
& \times \exp \left\{-\mathbf{Y}^{H}\left(\mathbf{X}_{D} \mathbf{F}_{L} \mathbf{R}_{h} \mathbf{F}_{L}^{H} \mathbf{X}_{D}^{H}+\sigma_{n}^{2} \mathbf{I}_{N}\right)^{-1} \mathbf{Y}\right\} .
\end{aligned}
$$

If $X_{k}$ is from a unitary constellation ${ }^{1}$, i.e., MPSK, $\mathbf{X}_{D}^{H} \mathbf{X}_{D}=$ $\mathbf{I}_{N}$. Hence $\operatorname{det}\left(\sigma_{n}^{2} \mathbf{I}_{N}+\mathbf{F}_{L}^{H} \mathbf{X}_{D}^{H} \mathbf{X}_{D} \mathbf{F}_{L}\right)$ is independent of $\mathbf{X}_{D}$. Maximizing (17) is equivalent to minimizing

$$
\begin{aligned}
g(\mathbf{X}) & =\mathbf{Y}^{H}\left(\mathbf{X}_{D} \mathbf{F}_{L} \mathbf{R}_{h} \mathbf{F}_{L}^{H} \mathbf{X}_{D}^{H}+\sigma_{n}^{2} \mathbf{I}_{N}\right)^{-1} \mathbf{Y} \\
& =\mathbf{X}^{T} \mathbf{Y}_{D}^{H}\left(\mathbf{F}_{L} \mathbf{R}_{h} \mathbf{F}_{L}^{H}+\sigma_{n}^{2} \mathbf{I}_{N}\right)^{-1} \mathbf{Y}_{D} \mathbf{X}^{*}
\end{aligned}
$$

where $\mathbf{Y}_{D}=\operatorname{diag}\left\{Y_{0}, \ldots, Y_{N-1}\right\}$ and $\mathbf{X}=$ $\left[X_{0}, \ldots, X_{N-1}\right]^{T}$. This semi-blind data detector (18) also incurs a phase ambiguity. Pilot symbols are thus still needed. When the data symbols $\mathbf{X}$ are estimated, the CIR can also be estimated using MMSE estimator as

$$
\hat{\mathbf{h}}=\left(\mathbf{F}_{L}^{H} \mathbf{F}_{L}+\sigma_{n}^{2} \mathbf{R}_{h}^{-1}\right)^{-1} \mathbf{F}_{L}^{H} \mathbf{X}_{D}^{H} \mathbf{Y} .
$$

If the channel remains constant in $K$ symbols, the channel estimate $\hat{\mathbf{h}}$ can be used to detect the remaining $K-1$ symbols.

\footnotetext{
${ }^{1}$ Note that the semi-blind data detector can also be generalized to nonunitary constellations.
}

The semi-blind data detector (18) needs the knowledge of $\mathbf{R}_{h}$ and $\sigma_{n}^{2}$, which may not be known perfectly in practice. However, $\mathbf{R}_{h}$ can be estimated using $\hat{\mathbf{R}}_{h}=\sum_{k=1}^{K} \hat{\mathbf{h}}_{k} \hat{\mathbf{h}}_{k}^{H} / K$ and $\sigma_{n}^{2}$ can be obtained via $\sum_{k=1}^{K}\left\|\mathbf{Y}_{k}-\hat{\mathbf{X}}_{k} \mathbf{F}_{L} \hat{\mathbf{h}}_{k}\right\|^{2} /(K N)$, where $\hat{\mathbf{h}}_{k}$ and $\hat{\mathbf{X}}_{k}$ are the estimated CIR and data symbols in the $k$-th symbol respectively. We can also show that the semi-blind detector (18) is robust to mismatch.

\section{B. Detection algorithms}

A straightforward approach to solve (18) is by exhaustively searching all the feasible candidates $\mathbf{X}$, which becomes impractical as $N$ becomes large. Instead, we suggest two algorithms to efficiently solve (18).

1) V-BLAST: The quadratic form (18) is similar to the cost metric in BLAST type MIMO systems. Therefore it can be solved by using V-BLAST detection algorithm [9]. The VBLAST ordering is to find the permutation matrix $\boldsymbol{\Pi}$ such that the $\mathrm{QR}$ decomposition of $\mathbf{H}=\mathbf{Q R}$ has the property that $\min _{1<i<N} r_{i i}$ is maximized over all column permutations. For $k=\bar{N}, N-1, \ldots, 1$, the algorithm chooses $\pi(k)$ such that

$$
\pi(k)=\underset{j \notin\{\pi(1), \ldots, \pi(k-1)\}}{\arg \min }\left\|\left(\mathbf{G}_{k}\right)_{j}\right\|^{2}
$$

where $\left(\mathbf{G}_{k}\right)_{j}$ is the $j$ th row of $\mathbf{G}_{k}, \mathbf{G}_{k}$ is the pseudo inverse of $\mathbf{H}_{k}$ and $\mathbf{H}_{k}$ denotes the matrix obtained by zeroing columns $\pi(1), \ldots, \pi(k-1)$ of $\mathbf{H}$. The $\mathrm{QR}$ factorization of $\mathbf{H}^{\prime}=\mathbf{H} \boldsymbol{\Pi}$ is denoted by $\mathbf{Q R}=\mathbf{H}^{\prime}$. Proceeding in the order $X_{N}, X_{N-1}, \ldots, X_{1}$ and assuming correct previous decisions, we can estimate $\mathbf{X}$ completely by cancelling interference successively. This sequential detection suffers from error propagation even with the above optimal ordering. Efficient implementations of V-BLAST require $O\left(N^{3}\right)$ computations.

2) Sphere decoder: SD generalizes the V-BLAST, where at each step, one considers all the $X_{k}$ 's that lie within a sphere of certain radius. SD has the important advantage over $\mathrm{V}$ BLAST in that it computes the exact ML solution. For a fixed radius and for a given lattice structure, the decoding complexity reduction of SD is usually significant, particularly in high SNR. However, for large $N$, which is case of the OFDM systems, the complexity of SD is high even in high SNR. The basic SD can be modified to first use Schnorr and Euchner [11] variant of SD (SESD). When a certain number of candidate points within the hypersphere have been found by $\mathrm{SD}$, the best point up to now can be output, which results in suboptimal detection. Note that the first point found by SESD is the V-BLAST solution. Therefore both the performance and complexity of the modified algorithm are between V-BLAST and SD.

\section{Simulation Results}

We consider a frequency-selective Rayleigh fading channel with $L=3$ and the Gaussian complex coefficients $h_{l}$ with mean power of $\sigma_{l}^{2}=E\left[\left|h_{l}\right|^{2}\right]=\sigma_{0}^{2} e^{-l / 5}$ for $l=1, \cdots, L$. An OFDM system with $\mathrm{N}=32$ subcarriers and binary phase shift keying (BPSK) is simulated. The carrier frequency is 


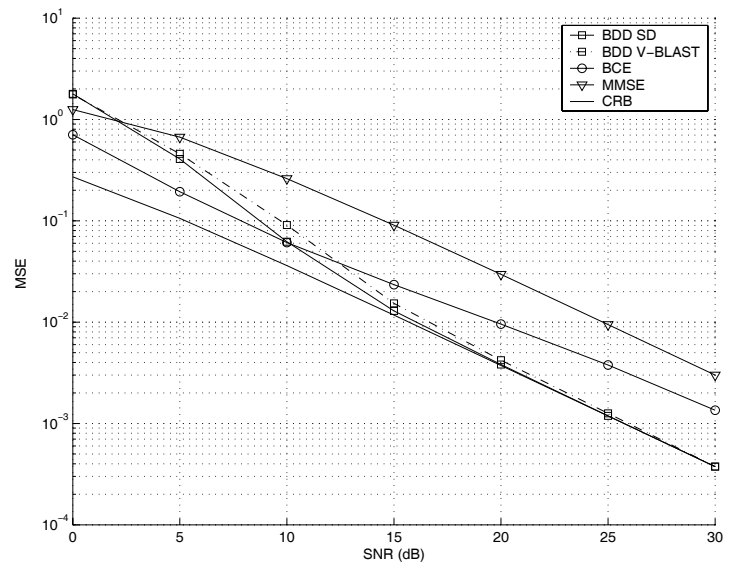

Fig. 1. MSE of an OFDM system with BPSK, $N=32$ and $N_{p}=4$.

$5 \mathrm{GHz}$ and the data rate is $500 \mathrm{kbps}$. Four pilot symbols are uniformly inserted into each OFDM symbol to solve the phase ambiguity. Both the semi-blind channel estimator (BCE) (12) and the semi-blind data detector (BDD) are tested on OFDM systems with the above simulation parameters.

Fig. 1 shows the mean square error (MSE) of CIR. The BDD first uses (17) to detect data symbols and then substitutes the detected data symbols into (19) to estimate the CIR. The performance of MSE of BDD with V-BLAST is almost the same as BDD with $\mathrm{SD}$ at $\mathrm{MSE}=10^{-2}$. They perform $4 \mathrm{~dB}$ better than the BCE and $9 \mathrm{~dB}$ better than the MMSE. The performance loss of $\mathrm{BCE}$ compared to $\mathrm{BDD}$ can be compensated by using the decision directed algorithm. However, with the same number of pilots, the BCE performs better than the MMSE estimator (14). The BCE has $5 \mathrm{~dB}$ gain over the MMSE at MSE $=10^{-2}$. This verifies the BCE (12) can use the side information embedded in the received signal to enhance channel estimation. The Cramér-Rao Bound (CRB) of the CIR is also shown. In the high SNR region, the BDD achieves the CRB.

The BER is compared in Fig. 2. The performance of onetap equalization with the CIR being perfectly known to the receiver (ideal case) is used as the benchmark. Both BCE and BDD performs close to the benchmark. Compared with it, the BCE has a $1 \mathrm{~dB}$ loss, the BDD with V-BLAST has a 0.6dB loss and the BDD with SD also has a $0.6 \mathrm{~dB}$ loss at $\mathrm{BER}=10^{-3}$. The performance of V-BLAST for BDD is comparable to that of SD in high SNR. Even in low SNR, the gap between SD and V-BLAST is within $0.5 \mathrm{~dB}$. The performance of MMSE channel estimator (14) is also shown. The BCE has a $1.6 \mathrm{~dB}$ gain over MMSE at $\mathrm{BER}=10^{-3}$. Though BDD performs better than $\mathrm{BCE}$, the performance loss using BCE is negligible and the complexity of BDD is high even using V-BLAST and SD. In a practical system, BCE is preferable.

\section{CONCLUSION}

We have developed a novel semi-blind channel estimator and a semi-blind data detector in OFDM systems. The semiblind channel estimator is derived by modelling the time

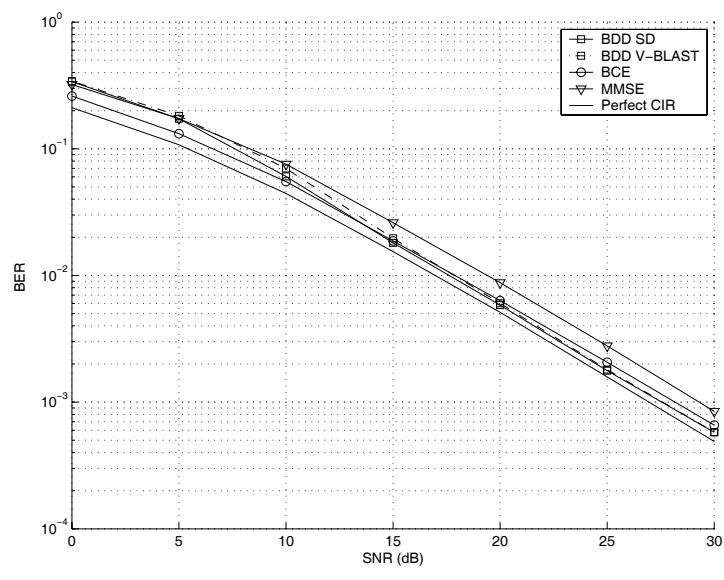

Fig. 2. BER of an OFDM system with BPSK, $N=32$ and $N_{p}=4$.

domain transmitted signal as Gaussian (the central limit theorem). Our semi-blind channel estimator, an enhanced LS estimator, is obtained by averaging the likelihood function over the CIR, resulting in a quadratic optimization problem. V-BLAST and SD have been used to efficiently detect data symbols. Simulation results show that our proposed channel estimator and data detector perform close to the ideal case. The detectors proposed in this paper may also be extended to MIMO-OFDM systems and OFDM over fast fading channels.

\section{REFERENCES}

[1] O. Edfors, M. Sandell, J.-J. van de Beek, S. Wilson, and P. Borjesson, "OFDM channel estimation by singular value decomposition," IEEE Transactions on Communications, vol. 46, no. 7, pp. 931-939, Jul. 1998.

[2] Y. Li, "Pilot-symbol-aided channel estimation for OFDM in wireless systems," IEEE Transactions on Vehicular Technology, vol. 49, no. 4, pp. 1207-1215, Jul. 2000

[3] B. Muquet, M. de Courville, and P. Duhamel, "Subspace-based blind and semi-blind channel estimation for OFDM systems," IEEE Transactions on Signal Processing, vol. 50, no. 7, pp. 1699 - 1712, July 2002.

[4] J. Heath, R.W. and G. Giannakis, "Exploiting input cyclostationarity for blind channel identification in OFDM systems," IEEE Transactions on Signal Processing, vol. 47, no. 3, pp. 848-856, Mar. 1999.

[5] S. Zhou and G. Giannakis, "Finite-alphabet based channel estimation for OFDM and related multicarrier systems," IEEE Transactions on Communications, vol. 49, no. 8, pp. 1402-1414, Aug. 2001.

[6] C. Li and S. Roy, "Subspace-based blind channel estimation for OFDM by exploiting virtual carriers," IEEE Transactions on Wireless Communications, vol. 2, no. 1, pp. 141-150, Jan. 2003.

[7] N. Chotikakamthorn and H. Suzuki, "On identifiability of OFDM blind channel estimation," in Proceedings of Vehicular Technology Conference, vol. 4, Sep. 1999, pp. 2358-2361.

[8] U. Fincke and M. Pohst, "Improved methods for calculating vectors of short length in a lattice, inlcuding a complexity analysis," Math. Computation, vol. 44, pp. 463-471, Apr. 1985.

[9] G. D. Golden, G. J. Foschini, R. A. Valenzuela, and P. W. Wolniansky, "Detection algorithm and initial laboratory results using the V-BLAST space-time communication architecture," Electronics Letters, vol. 35, no. 1, pp. 14-15, Jan. 1999.

[10] H. Ochiai and H. Imai, "On the distribution of the peak-to-average power ratio in OFDM signals," IEEE Transactions on Communications, vol. 50, no. 1, pp. 89 - 101, Jan. 2001.

[11] C. P. Schnorr and M. Euchner, "Lattice basis reduction: Improved practical algorithms and solving subset sum problems," Math. Programming, vol. 66, pp. 181-191, 1994. 